\title{
Claudia Agnes Müller (2015): Forschendes Theater. Chancen und Potential im Kontext von Spracherwerb, transkultureller Landeskunde und studentischer Performance.
}

Berlin: Universitätsverlag der TU - ISBN 978-3-7983-2740-5 (print); ISBN 978-3-7983-2741-2 (online)

\section{Anne Steiner}

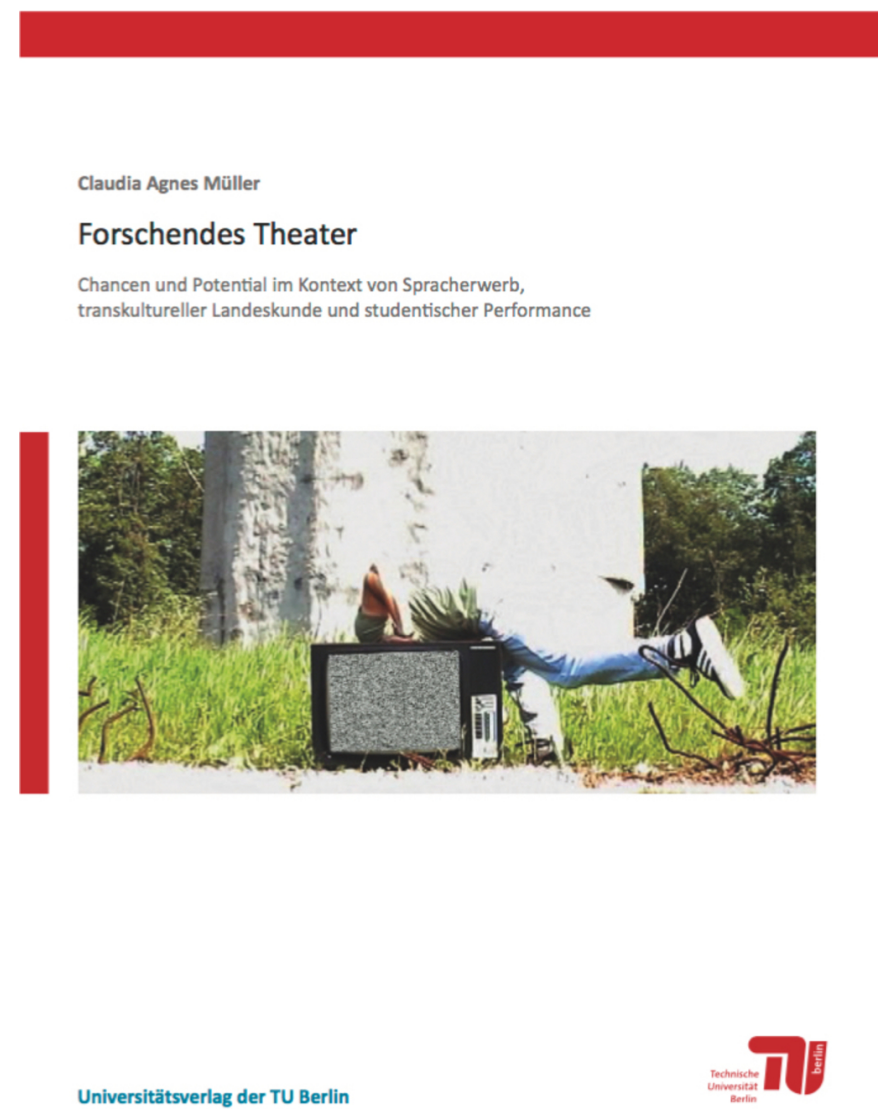

Forschendes Theater hat sich als eine besondere Spielart des Theaters etabliert. Die Zahl der Performancegruppen, die gesellschaftliche Realität erforschen und - oft gemeinsam mit ihrem Publikum - soziale Wirklichkeit(en) ausloten und mit biographischem Material experimentieren, scheint in den 
letzten Jahren explodiert zu sein. Angekommen ist das forschende Theater auch in der Wissenschaft, Tagungen wie „Forschendes Theater in sozialen Feldern“ an der FH Dortmund im November 2016 setzten sich bereits mit dem Thema auseinander. Und auch in der Schule wird forschendes Theater betrieben schon 2015 widmete sich das bundesweite Festival „Schultheater der Länder" diesem Thema.

Claudia Agnes Müller knüpft an diese Entwicklung an. Sie stellt in ihrem Buch aktuelle Projekte und Gruppen vor, die dem forschenden Theater zugerechnet werden, berichtet über eigene Theaterforschungsprojekte mit Studierenden in verschiedenen Ländern und zeichnet wichtige Entwicklungslinien des forschenden Theaters nach. So spannend sich das alles auch liest - welches Potential die beschriebenen Theaterprojekte für den (Fremd)Spracherwerb haben, wird trotz Ankündigung im Titel nicht erläutert - der/die Leser/in bleibt deshalb nach der Lektüre etwas ratlos zurück. Dass Müller am Ende ihrer Einleitung kurz die Aspekte aufzählt, die ihr Titel zwar erwarten lässt, die aber in ihrem Band leider „keinen Platz“ (10) finden können („Es wird keine systematische Darstellung der Beeinflussung der Sprachentwicklung bei Deutschlernenden, die an einem Theaterprojekt beteiligt waren, geben. [... ] Es wird [...] nicht explizit auf interkulturelle Theaterarbeit eingegangen [...]." (10)), verkleinert die Irritation nicht.

Unbefriedigend ist auch ein anderer Aspekt: Was dieses „forschende“ Theater nun genau ist, wird nicht klar definiert. Es finden sich zwar in vielen Kapiteln wie beiläufig Zuschreibungen und Charakterisierungen, etliche davon sind jedoch so allgemein gehalten, dass sie im Grunde auf jedes Theaterprojekt anwendbar sind. Wird beispielsweise „alternatives Wissen zu generieren, das sich mit den Kategorien der Wissenschaft niemals beschreiben ließe“ (15) als Ziel forschenden Theaters umrissen, dann ist das wenig erhellend, da doch jegliche Kunst in Produktion und Rezeption Erfahrungen ermöglicht und damit Wissen generiert, das von der Wissenschaft allein nicht hätte bereitgestellt werden können - und selbst die Rezeption einer traditionell eingerichteten Schauspielaufführung eines kanonischen Dramentextes birgt ästhetische Erfahrungsmomente, aus denen individuell neue Wissensbestände generiert werden können. Eine zusammenfassende Definition des forschenden Theaters, die über solch Allgemeines hinausgeht, sucht man leider vergeblich; der Begriff des Forschens bleibt ein vages Sammelsurium. Ob dies daran liegt, dass Müller sehr gut informierte, mit reichlichem Vorwissen ausgestattete Leser/innen erwartet, oder daran, dass es ,das' forschende Theater vielleicht gar nicht gibt, wäre zu diskutieren.

Dass Müller nicht frei von Widersprüchen argumentiert, ist schließlich ein weiterer Kritikpunkt an ihrer Studie. Wenn beispielsweise tatsächlich so pauschal und absolut immer gelten müsste, was Müller mit „[d]er Ansatz des forschenden Theaters verzichtet konzeptionell auf jede Form einer Kombination von vorgefertigten Elementen“ (16) zunächst konstatiert, dann dürfte sie Vorschläge wie diesen für forschende Theaterprojekte mit Studierenden nicht unterbreiten: „Mit ,Schubläden' findet sich bei She She Pop ein weiteres 
Projekt, das einen reizvollen Ansatzpunkt für eine Adaption oder sogar für ein direktes Reenactment bietet.“ (143) Reenactment, dieses möglichst getreue und authentische Nachspielen oder Nachstellen konkreter Ereignisse, bedient sich ganz bewusst und gewollt vorgefertigter und bereits gegebener Elemente und tut damit das, was forschendes Theater laut Müller eben gerade nicht will und nicht soll.

Lesenswert ist dieses Buch dennoch. Seine Leistung liegt darin, kenntnisreich über zahlreiche forschende Theaterprojekte, ihre Arbeitsweisen und Ergebnisse, aber auch ihre Vorgänger und Vorläufer und deren Arbeit zu informieren, unter Rückgriff auf die einschlägige Forschungsliteratur einen detaillierten Einblick in verschiedene Facetten des künstlerischen Forschens und die Geschichte und Grundlagen forschenden Theaters zu geben und dabei immer wieder auch interessante Überlegungen zur Arbeit mit studentischen Laiengruppen einfließen zu lassen, die dem/der Leser/in helfen, die Ansätze professioneller Performer und Theaterforschender für die eigene theaterpädagogische Arbeit mit Laien jeglichen Alters zu reflektieren.

Da es ihr um die Arbeit mit studentischen Laien geht, die sich theaterforschend mit fremder und eigener kultureller, gesellschaftlicher, sozialer, sprachlicher ... Wirklichkeit auseinandersetzen sollen, geht Müller zunächst kritisch auf einige wichtige theaterpädagogische Ansätze ein (11-33). Sie beschäftigt sich mit dem Theaterlabor von Otto, der theaterpädagogischen Inszenierung von Hilliger, dem biografischen Theater von Plath und dem Psychodrama von Moreno und zeigt auf, in welchem Zusammenhang diese mit forschendem Theater stehen und welche Gemeinsamkeiten und Unterschiede sich in der Arbeit mit Laien und mit professionellen Spieler/innen zeigen. Nach einem kurzen Exkurs, einem Erfahrungsbericht zu einem eigenen Theaterprojekt mit Studierenden, bei dem sie einen forschenden Ansatz verfolgte (35-48), zeichnet Müller die „Entwicklungslinien des forschenden Theaters“ (49-88) nach und setzt sich dafür exemplarisch Grotowski, Brook, Boal und Schlingensief auseinander, deren Ansätze, Arbeitsweisen und „Forschungsmethoden“ sie ebenso vergleichend in den Blick nimmt wie deren Umgang mit den Spieler/innen und dem Publikum. Daran anschließend reflektiert sie die Kontaktmöglichkeiten von Kunst und Wissenschaft (89-113) und argumentiert nachvollziehbar, welchen Beitrag für die Generierung von Wissen sie jeweils leisten können: Wissenschaft ist für Müller „eine konsequente Strategie der Problembeseitigung“ (91), Kunst dagegen „sucht nicht nach Lösungen, sie vertieft das Bewusstsein für Probleme." (91-92) Beide jedoch ,sind in ihrem Bestreben nach wirklich neuer Erkenntnis vor allem eine bestimmte, auf das Unvorhersehbare ausgerichtete Praxis“ (97) mit gemeinsamer Herkunft und sowohl divergierenden als auch konvergierenden Wegen.

Den Hauptteil ihrer Studie widmet Müller der lebendigen und vielfältigen Szene des forschenden Theaters (115-299), das Erinnerungsarbeit ebenso einbeziehen kann wie die Simulation von Zukunft oder die Auseinandersetzung mit ökonomischen Gesetzmäßigkeiten der Gegenwart. Sie beschreibt und analysiert Arbeitsweisen und Zielsetzungen verschiedenster Performancegruppen 
und künstlerischer Kollektive (wie TWO FISH, She She Pop, Rimini Protokoll, um nur einige zu nennen) und die Ergebnisse forschender Theaterarbeit und an diese angrenzender Strömungen, die sich in ganz unterschiedlichen Projekten (wie beispielsweise „Das Botanische Langzeittheater") präsentieren. So gelingt es Müller, die Vielfalt an Fragestellungen, die theaterforschend erkundet werden, und an Vorgehensweisen und Methoden, die dabei verfolgt werden, aufzudecken und für die Arbeit mit Laien zu diskutieren. Wie schade, dass dabei der behauptete transkulturelle Zugriff nicht deutlich wird und Aspekte des Spracherwerbs und der Sprachvermittlung nicht intensiv beleuchtet werden. 